\title{
LA ORIENTACIÓN DE LAS IGLESIAS PRERROMÁNICAS DE GALICIA: ANÁLISIS Y RESULTADOS PRELIMINARES
}

\author{
A. CÉSAR GONZÁLEZ-GARCÍA ${ }^{(1)}$
}

Resumen:

\begin{abstract}
Se muestran los resultados de la labor de medida de las orientaciones de los ábsides y cabeceras de 25 iglesias prerrománicas analizadas hasta el momento en Galicia. Si bien el trabajo es aun preliminar ya se pueden extraer un número de interesantes resultados y conclusiones. Las iglesias de nuestra muestra presentan características comunes a las de los templos de la misma época del resto de la Península. Aparece una tendencia generalizada a mostrar una orientación de la iglesia hacia puntos ligeramente hacia el norte respecto al equinoccio astronómico. Esta tendencia es consistente con un uso de la salida del sol el 25 de Marzo para la época de construcción como guía para tal fin. Un caso paradigmático de tal uso y de su pervivencia en el tiempo puede ser la propia catedral de Santiago. Existen excepciones a tal regla, siendo las más características la presencia de orientaciones a poniente entre las iglesias identificadas como las más antiguas. Es interesante que en algún caso el uso de las orientaciones permite ratificar la posible conexión entre la iglesia de San Xes de Francelos con el grupo de iglesias asturianas.
\end{abstract}

Palabras clave: Iglesias Prerrománicas, Orientación, Astronomía en la Cultura, Calendario, Ritual Cristiano, Galicia

\begin{abstract}
:
The orientation of pre-Romanesque churches in Galicia: analysis and preliminary results We present the results of the on-going effort to measure the orientation of a comprehensive number of churches in Galicia. We have measured the orientation of the apse of 25 churches so far. Although the results are still preliminary we can obtain a number of interesting conclusions. The churches in our sample present a similar trend to those of the same period recently measured in the rest of the Iberian Peninsula. There is a general tendency towards orientations slightly north of due equinoctial east. This tendency is consistent with a use of sunrise on March 25th at the time of construction to orientate the building. In this respect, the Cathedral of Santiago may be a paradigmatic case. There are exceptions to this rule and the most interesting would be that early churches do appear to be facing westwards instead of eastward. Finally, the orientation of San Xes de Francelos allows identification with the group of churches of Asturias.
\end{abstract}

Keywords: Pre-Romanesque Churches, Orientation, Astronomy in Culture, Calendar, Christian Ritual, Galicia

Received: 14 November, 2014; Accepted: 23 March, 2015

\section{INTRODUCCIÓN}

El estudio de la orientación de las iglesias cristianas ha ganado recientemente un nuevo auge en la literatura, ya que este es un factor representativo clave de su arquitectura. De acuerdo con los textos de los primeros escritores y apologetas cristianos, las iglesias deben estar orientadas de un modo particular, de forma que el sacerdote dirija sus oraciones hacia el este durante los servicios. Así lo apuntan Orígenes, al igual que Clemente de Alejandría y Tertuliano y, finalmente, se hizo oficial en el Concilio de Nicea (325). San Atanasio de Alejandría, también en el siglo IV, dice que el sacerdote y los participantes deben situarse mirando hacia el este, donde está Cristo, el Sol de Justicia, que brillaría en el final de los tiempos (ecclesiarum situs plerumque erat talis, ut fideles facie Altare versa orientem Solem, symbolum Christi qui est sol Iustitia et lux mundi interentur [...]). Véase Vogel (1962) para un análisis a fondo de las fuentes y los métodos de orientación.

Si nos fijamos en si estos preceptos se siguie- ron, no todas las primeras basílicas cristianas fueron construidas con el ábside, o la cabecera de la iglesia mirando hacia el este. En este sentido, Delgado-Gómez (2006) indica que de las 20 primeras basílicas cristianas construidas durante la época de Constantino y sus sucesores en Roma, Jerusalén, Constantinopla y el Norte de África, 18 están situadas aproximadamente en la línea este-oeste, 11 de las cuales tienen el ábside orientado hacia el oeste. Sin embargo, es interesante destacar que en esos casos el sacerdote reza de todos modos hacia el este, ya que el altar se encuentra entre él y los fieles.

Entre los siglos tercero y séptimo las Constituciones Apostólicas indican claramente que las iglesias deben construirse mirando hacia el este, refinando esta prescripción (Const. Apost., II, 7). En el siglo quinto, Sidonio Apolinar y Paulino de Nola indican que el ábside debe mirar hacia el este, hacia el equinoccio, lo cual fue confirmado por el Papa Virgilio e Isidoro de Sevilla en su Etimologías (XV, 4; MCCLUSKEY 1998). Honorio Augustodunense nos lo ratifica durante la plena Edad Me-

${ }^{(1)}$ Instituto de Ciencias del Patrimonio, Incipit. Consejo Superior de Investigaciones Científicas. San Roque 2, 15705 Santiago de Compostela. 
dia (siglos XI-XII [...] ecclesiae ad orientem vertuntur oritur ubi sol [...]), así como otros autores como Guillermo Durando (siglos XII-XIII, [...] versus orientem, hoc est, versus Ortum aequinoctialem solis, nec vero aestivale Solstitium [...]), lo que indica claramente la dirección a seguir, el equinoccio, evitando el solsticio.

Esta orientación hacia el este tiene un claro simbolismo como ya hemos indicado. Es en esa dirección por donde sale el sol y por lo tanto marca la dirección de la que habrá de venir Cristo, que se levantará como el Sol de la Justicia a partir del Día del Juicio. Además, era más o menos la zona hacia la que los cristianos del Occidente Mediterráneo estarían enfrentando Jerusalén (MCCLUSKEY 2004, 2010).

Sin embargo, estos requisitos siguen siendo un tanto ambiguos, ya que no está clara la definición sobre cómo construir la iglesia hacia el este. Las fuentes apuntan a la necesidad de orientar el ábside al este hacia el equinoccio, pero uno puede preguntar a qué equinoccio debe estar mirando el ábside. Según menciona McCluskey (2004), hay varias formas de obtener un equinoccio: el equinoccio de primavera romano ocurría el 25 de marzo, mientras que el griego era el 21 de marzo, como se refleja en el Concilio de Nicea. Pero se pueden dar otras definiciones, como la entrada del sol en el signo de Aries o el equinoccio de otoño ( 24 de septiembre o el 21 para el equinoccio tradicional romano o griego respectivamente). Cada una de estas definiciones (RUGGLES 1999, GONZÁLEZ-GARCíA \& BELMONTE 2006) resulta en fechas diferentes, y por lo tanto en orientaciones ligeramente distintas entre sí. Otro punto importante a considerar es el uso del calendario Juliano durante la Edad Media y buena parte del Renacimiento. Si nos concentramos en una definición cultural particular de equinoccio, es decir, en una fecha determinada, por ejemplo el 25 de marzo, la naturaleza del calendario juliano (cuya duración es ligeramente mayor que la del año trópico) haría que dicha fecha se desplace con respecto a las estaciones del año, lo que se reflejaría en un cambio sistemático en la orientación con los siglos.

Es común en la literatura que aparezcan otras posibilidades para obtener la orientación de la iglesia, tales como el uso de la salida del sol en el día del santo patrón de la iglesia, la posición del sol en el día que comienza la construcción de la iglesia o en algunas fiestas importantes específicas para la comunidad. Con respecto a la orientación de las iglesias hacia la salida del sol en día de la festividad del santo patrón, esta posibilidad no aparece en los primeros escritos y hasta bien entrada la Edad Media no hay respaldo escrito de tal reclamación. Hasta la aparición de las órdenes religiosas a finales de la época medieval, no encontramos tal tendencia. Por ejemplo, Johnson (1912), aunque menciona la idea de orientar las iglesias "hacia el punto de la salida del sol que correspondía al día del patrón a quien está dedicada la iglesia" también indica que esta idea no es detectable antes de mediados del siglo XVII, en concreto en un manuscrito donde Silas Taylor dice: “... he experimentado (sic) algunas iglesias y he encontrado la línea para que apunte a esa parte del horizonte donde nace el sol en el día del santo a quien está dedicada la iglesia". Algo similar lo describe el poeta británico William Wordsworth en un poema escrito en 1824 pero publicado varios años después, con motivo de la fundación de la Capilla de Rydal. Chauncey (1700), sin embargo señala que: "Uno de los extremos de cada Iglesia debe apuntar al lugar donde el sol salió en el momento de la fundación de la misma... y por la medida de estas iglesias, se sabe en qué momento del año se fundaron los cimientos". Es importante señalar que todas estas informaciones son más bien tardías en la historia de la edificación de iglesias.

\section{EL ESTUDIO DE LA ORIENTACIÓN DE IGLE SIAS MEDIEVALES EN EUROPA}

En este contexto, el estudio de las orientaciones de las iglesias cobra un especial interés por aportar datos para poder comparar con las diversas alternativas que nos ofrecen las fuentes.

El estudio de la orientación de las iglesias medievales de Europa, junto con las pirámides de Egipto y los monumentos megalitos, es uno de los temas más antiguos que se han trabajado en $\mathrm{Ar}$ queoastronomía (NISSEN 1906). González-García (2015) muestra que la orientación hacia el Este es seguida de manera consistente en toda Europa, al menos durante la Edad Media, como se muestra en la Figura 1 (panel A). Esta figura muestra datos para un total de 560 iglesias de toda Europa. Todas las áreas consideradas en su estudio siguen este patrón con un pico predominante claro centrado en torno al equinoccio (el equinoccio astronómico vendría indicado por un valor cercano a 0 en este diagrama). En muchos casos, sobre todo en Europa occidental, este máximo está desplazado ligeramente hacia el norte (valores positivos en la Fig. 1A) en relación con el valor del equinoccio astronómico, quizás indicando un uso de las fechas específicas para el equinoccio (tal vez 25 de marzo).

Respecto a orientaciones relacionadas con las festividades de los santos patronos, GonzálezGarcía (2015) indica que es posible que aparezcan en algunas zonas de Alemania y tal vez en Inglaterra y Francia, pero destacando que estos edificios son en general las iglesias monásticas románicas y las catedrales góticas. Un caso interesante y bien documentado ocurre en Eslovenia, donde Čaval (2009) encontró evidencias de un grupo con un número significativo de iglesias románicas cuyas orientaciones aparecen relacionadas con la fiesta de la Cátedra de San Pedro, una festividad que dicha autora encontró particularmente importante en la época de construcción de esas iglesias. McCluskey (2004) indica que algo similar ocurre en Inglaterra para la orientación de las iglesias románicas del centro de este país, don- 
de quizás los santuarios con advocaciones marianas pueden seguir una orientación hacia fechas de fiestas marianas de forma complementaria a la orientación predominante hacia el este. Curiosamente, una excepción a la regla de las orientaciones hacia el este se produce en el norte de África, donde hasta el siglo VII se construyeron iglesias con orientaciones hacia poniente. Hay un número de iglesias con orientaciones hacia el oeste, una costumbre habitual en los primeros tiempos del cristianismo, como se señaló anteriormente (ESTEBAN et al. 2001, BELMONTE et al. 2007). González-García (2015) también ha demostrado que la mayoría de estas iglesias están dispuestas dentro del rango de orientaciones compatibles tanto con la salida como con la puesta solares, con concentraciones en los equinoccios y solsticios, que podrían dar pistas sobre el proceso de cristianización de la región.
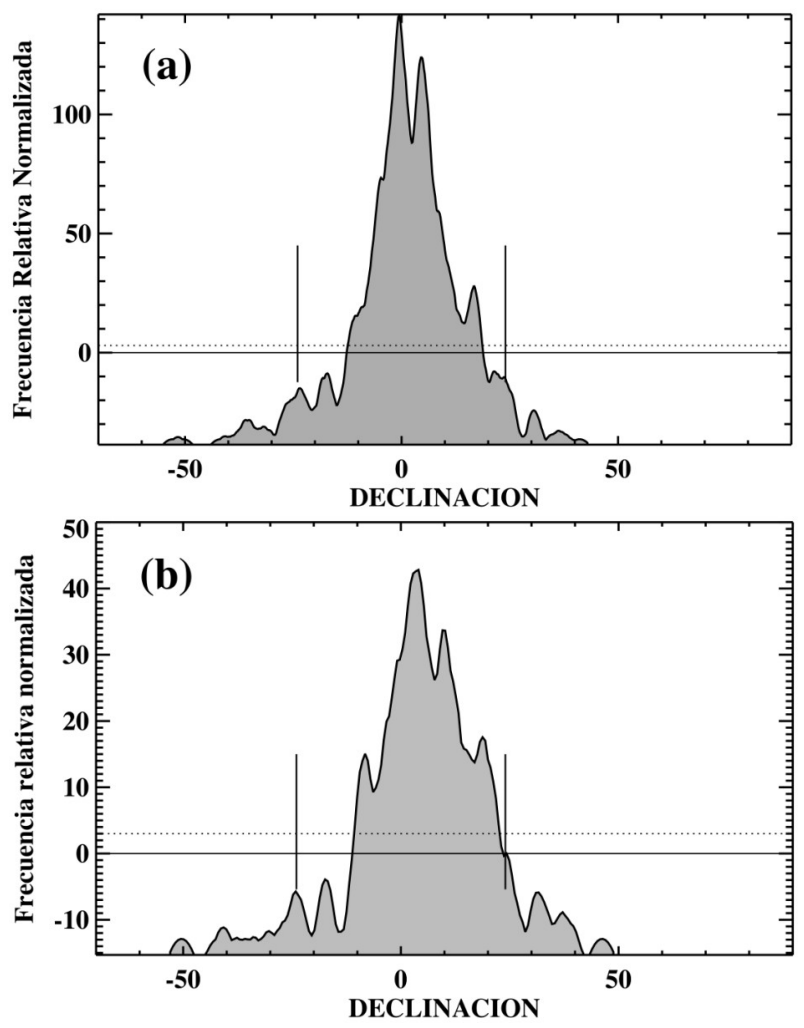

Fig. 1. Histogramas de declinación de las Iglesias de Europa y prerrománicas de la Península Ibérica. El panel (a) muestra datos de más de 560 iglesias medievales de toda Europa. Las líneas sólidas verticales indican los extremos solares (entre $-23.5^{\circ}$ y $23.5^{\circ}$ ), mientras que el equinoccio astronómico tiene declinación $0^{\circ}$. Esta figura muestra que la mayoría de las Iglesias están orientadas aproximadamente al este con una tendencia a evitar los solsticios. El panel (b) muestra el histograma de declinación de 167 iglesias prerrománicas de la Península Ibérica. La mayor concentración ocurre para declinaciones ligeramente al norte del equinoccio astronómico. Existen otras acumulaciones a declinaciones de $\sim 10^{\circ}$, $-10^{\circ}$ and $20^{\circ}$. Estas orientaciones se pueden relacionar con algunas festividades cristianas, como la última que se podría corresponder con la fecha del día de Santiago Apóstol el 25 de Julio.

Fig. 1. Declination histograms for the measured churches in Europe and the pre-Romanesque churches in the Iberian Peninsula. Panel (a) shows data for more than 560 medieval churches all over Europe. Solid vertical lines indicate the solar extremes (between $-23.5^{\circ}$ and $23.5^{\circ}$ ), while astronomical equinox is at declination $0^{\circ}$. This figure shows that most churches have orientations close to the equinox with a tendency to avoid the solstices. Panel (b) shows the declination histogram for 167 pre-Romanesque churches in the Iberian Peninsula. The largest concentration appears for declinations slightly north of the astronocimal equinox. There are other accumulations at declinations $\sim 10^{\circ},-10^{\circ}$ and $20^{\circ}$. These orientations might be related to several Christian festivities. It is remarkable the last one, that may correspond to the festivity of Sain James on July 25 th.

En la Península Ibérica y en los dos archipiélagos (Baleares y Canarias) la cuestión de la orientación de las iglesias se ha investigado tradicionalmente de manera muy general, aunque hay informes de determinados eventos de iluminación dentro de algunas iglesias románicas en momentos especiales como el equinoccio (tales como los fenómenos que ocurren en Santa Marta de Tera o San Juan de Ortega, en las provincias de Zamora y Burgos, respectivamente). Esto ha conducido a reclamaciones sin fundamento con respecto a la posible causa de la desviación de algunas iglesias con respecto a la orientación estándar hacia el este (véase, por ejemplo GODOY-FERNÁNDEZ 2004). 
Digno de mención es el trabajo de Pérez-Valcárcel (1998) quien ha investigado la orientación de 187 iglesias románicas del Camino de Santiago, pero sus datos no incluyen la medición de la altura angular del horizonte, algo por desgracia muy común en muchos estudios europeos. Sin embargo, lo que sí parece claro es que se puede descartar una relación general entre la orientación de estas iglesias y la salida del sol en la fecha del santo patrón de la iglesia.

González-García y Belmonte (2014a) y González-García et al. (2015) dedican una especial atención a la época de las iglesias asturianas y su interacción con el poder musulmán dominante en el sur de la península. Específicamente, encontraron que las 13 iglesias que aún existen de ese periodo poseen una orientación canónica, con el ábside orientado hacia la mitad oriental del horizonte, aunque generalmente desviado de forma característica varios grados al norte del este. Por otra parte, los autores estudiaron también la orientación de las mezquitas de Al-Andalus, cuya qibla debería haber estado orientada hacia la Meca y por lo tanto, dadas las latitudes de Al-Andalus, deberían haber tenido orientaciones compatibles con las alineaciones canónicas de las iglesias hacia el oriente. Sin embargo las mezquitas siguieron un patrón peculiar. Por un lado, un número de mezquitas se orienta hacia el SE, mientras que otras siguen la qibla de la Gran Mezquita de Córdoba al S-SE, ambas disposiciones permitidas por el Islam en determinadas circunstancias. Así, parecería que los musulmanes en Al Andalus habrían 'evitado' orientaciones que pudieran confundir sus templos con las iglesias, mientras que los santuarios asturianos tienden a evitar las orientaciones que confunden estos templos con mezquitas. Este es un excelente ejemplo de la interacción de la religión, el poder y la astronomía.

Curiosamente, (ver GANGUI et al. 2015) las orientaciones de las iglesias en Lanzarote desde el período de la conquista castellana en el siglo XV hasta el siglo XIX, muestran que la mayoría de las iglesias siguen la orientación canónica. Sin embargo, un número significativo de ellas tiene orientaciones dictadas por una razón prosaica para evitar los vientos dominantes. Así, vemos que, en circunstancias excepcionales, los patrones canónicos pueden ser alterados.

García-Quintela et al. (2014) han investigado la introducción del cristianismo en el noroeste de la Península y la posible sustitución de cultos indoeuropeos (Celtas) por los cristianos mediante la introducción de lo que ellos llaman un "paisaje mártir" a través de la orientación de las iglesias y la cristianización de su entorno y la creación de mitos que los canalizan, modificado o reemplazado potenciales cultos paganos.

Finalmente, González-García \& Belmonte (2015) han medido la orientación de un total de 167 iglesias construidas antes de 1086 en la Península Ibérica y Baleares. El análisis estadístico muestra un patrón de orientación con una clara tendencia a orientarse según el equinoccio canónico del 25 de marzo (Figura 1, panel B). Es interesante que estos autores muestran que tal tendencia es consistente a lo largo de los casi 8 siglos que cubre su muestra y que queda reflejado en una variación secular de ese máximo principal hacia posiciones más hacia el norte con el tiempo, justo lo que cabría esperar que ocurriría con orientaciones hacia el 25 de marzo según el calendario Juliano. Hay otros grupos de orientaciones específicas para cada período, siendo el más interesante el grupo de orientación hacia la salida del sol el día de Santiago, que aparece para iglesias construidas después del descubrimiento de la tumba del Santo en el siglo noveno.

\section{MÉTODO}

Los valores medidos en nuestro trabajo de campo son el azimut y la altura del horizonte en la dirección de ese azimut. Los datos de Orientación presentados en la Tabla 1 fueron obtenidos en nuestro trabajo de campo utilizando brújulas de alta precisión y clinómetros. Los datos de las brújulas fueron debidamente corregidos de declinación magnética. Las mediciones de campo incluidos en la Tabla 1 tienen un error promedio de $1 / 2^{\circ}$ en azimut y $1 / 2^{\circ}$ en la altura del horizonte, que se traduce en un error $\sim^{3} / 4^{\circ}$ en declinación. Una serie de medidas se obtuvieron mediante imágenes de satélite o de ortofotos y planos. Estas se indican en la Tabla 1 en caracteres en cursiva. En estos casos particulares, la altura angular del horizonte se ha calculado desde el sitio $<$ heywhatsthat.com $>$ confirmándolo con las capacidades de Google Earth. Los diferentes errores introducidos en estos procedimientos aumentan hasta alrededor de $11^{\circ}$ en declinación. Estos errores se tratan por separado en la construcción de los histogramas, considerando un paso de banda del doble del error para cada una de estas entradas particulares.

La hipótesis de partida es que la orientación de las iglesias puede venir determinada por el orto solar en algún momento particular del año, ya sea este determinado por cuestiones cultuales o bien por motivos prácticos. Queremos así ver si esa orientación nos puede ayudar a determinar si las iglesias prerrománicas en Galicia se construyen teniendo en cuenta el este (y en tal caso, qué tipo de 'este' se está usando) o si existen consideraciones tales como el orto en el día del santo patrón.

Esta orientación se define como la línea perpendicular al ábside o cabecera de la iglesia, considerando desde el interior hacia la cabecera, bajo la hipótesis de que el altar principal estaría situado en esa posición y el sacerdote y los fieles de forma general se situarían mirando en tal dirección.

Hay que tener en cuenta que las medidas que hemos tomado, azimut y altura son dos coordenadas locales. Es decir, para un concreto lugar nos darán un valor para el orto solar determinado (dos 
Tabla 1. Datos de las 25 iglesias prerrománicas medidas hasta el momento en Galicia. Las dos primeras columnas dan el sitio y la iglesia medida. Las cuatro siguientes su localización en latitud $(\phi)$ y longitud $(\lambda)$. Las dos siguientes son los datos de su orientación en grados respecto al norte geográfico (Azimut-A) y la altura del horizonte (h) en esa dirección desde el ábside de la iglesia. La siguiente columna es la declinación astronómica correspondiente a esa orientación $(\delta)$. Las dos últimas columnas dan las fechas correspondientes de salida (o puesta) del sol en la dirección del azimut y las fechas de celebración de los santos de advocación de las iglesias.

Table 1. Data for the 25 pre-Romanesque churches measured so far in Galicia. The first two columns indicate the location and name of the church. The next four columns give the latitude $(\phi)$ and longitude $(\lambda)$. The next two provide the orientation data in degrees from true north (azimuth-A) and the altitude of the horizon (h) in the direction of the church's apse. The next colum gives the astronomical declination $(\delta)$ and the last two give the corresponding dates of sunrise (or sunset) in that direction and the dates for the celebration of the Patron Saints of the churches.

\begin{tabular}{|c|c|c|c|c|c|c|c|c|c|c|}
\hline Sitio & Iglesia & $\phi\left({ }^{\circ}\right)$ & (') & $\lambda\left({ }^{\circ}\right)$ & (') & $\mathbf{A}$ & h & $\delta$ & Fecha Gr. & Fecha Santo \\
\hline Bóveda & Sta.Eulalia & 42 & 58 & 7 & 41 & $931 / 4$ & 0 & -2.9 & $13 / 3-1 / 10$ & $12 / 2-10 / 12$ \\
\hline Augasantas & $\begin{array}{l}\text { St.Mariña/ } \\
\text { Ascensión }\end{array}$ & 42 & 14 & 7 & 47 & $119^{3 / 4}$ & $61 / 2$ & -16.9 & $1 / 2-10 / 11$ & $\begin{array}{l}18 / 7 \\
\text { movible }\end{array}$ \\
\hline Ouvigo & S.Verísimo & 42 & 0 & 7 & 45 & $288^{1 / 4}$ & $2 \frac{1}{2}$ & 15.0 & $1 / 5-12 / 8$ & $1 / 10$ \\
\hline Cidadela 1 & |----------- & 43 & 5 & 8 & 2 & 106 & $2 \frac{1}{2}$ & -10.0 & $22 / 2-19 / 10$ & \begin{tabular}{|l|}
--------- \\
\end{tabular} \\
\hline Cidadela 2 & 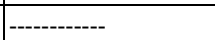 & 43 & 5 & 8 & 2 & 105 & $2 \frac{1}{2}$ & -9.3 & $24 / 2-17 / 10$ & |-------- \\
\hline Panxón & S. Xoan & 42 & 8 & 8 & 49 & $951 / 2$ & $31 / 2$ & -1.9 & $16 / 3-28 / 9$ & $24 / 6-27 / 12$ \\
\hline Ambía & Sta.Eufemia & 42 & 3 & 7 & 42 & 89.8 & 5 & 3.4 & $29 / 3-14 / 9$ & $20 / 3$ \\
\hline Bande & Sta. Comba & 41 & 58 & 8 & 0 & $91 \frac{11 / 4}{4}$ & 2 & 0.2 & $21 / 3-22 / 9$ & $17 / 9$ \\
\hline Francelos & S. Xes & 42 & 16 & 8 & 9 & $701 / 2$ & 2 & 15.5 & $2 / 5-10 / 8$ & $25 / 8$ \\
\hline Retorta & Sta. Cruz & 43 & 1 & 7 & 41 & $87^{3 / 4}$ & 2 & 2.8 & $28 / 3-16 / 9$ & $3 / 5$ \\
\hline Berbetouros & S. Miguel & 42 & 55 & 7 & 48 & $83 \frac{1}{2}$ & $01 / 2$ & 4.8 & $2 / 4-11 / 9$ & $29 / 9$ \\
\hline Santiago & Catedral & 42 & 52 & 8 & 32 & 86 & $01 / 2$ & 2.9 & $28 / 3-15 / 9$ & $25 / 7$ \\
\hline Santiago & Corticela/Sta. María & 42 & 52 & 8 & 32 & 79 & $1 \frac{1}{4}$ & 8.6 & $12 / 4-1 / 9$ & $1 / 1-19 / 5-15 / 8-8 / 12$ \\
\hline Pambre & Castillo/S. Pedro & 42 & 51 & 7 & 57 & $963 / 4$ & $1 \frac{1}{2}$ & -4.2 & $10 / 3-4 / 10$ & $29 / 6$ \\
\hline Pontecesures & S. Xulian & 42 & 43 & 8 & 39 & $85^{3 / 4}$ & 13 & 11.8 & $21 / 4-22 / 8$ & $9 / 1(7 / 1)$ \\
\hline Valga & Sta. Comba Louro & 42 & 41 & 8 & 41 & $83 \frac{1 / 4}{4}$ & $4 \frac{1}{2}$ & 7.9 & $10 / 4-2 / 9$ & $17 / 9$ \\
\hline Valga & S. Mamede & 42 & 41 & 8 & 37 & $881 / 2$ & 7 & 5.8 & $4 / 4-8 / 9$ & $17 / 8$ \\
\hline Viñao & S. Pedro & 42 & 22 & 7 & 59 & $901 / 2$ & 9 & 5.6 & $4 / 4-8 / 9$ & $29 / 6$ \\
\hline Fontefría & S. Xiao & 42 & 22 & 7 & 56 & $73^{3 / 4}$ & 1 & 12.3 & $23 / 4-20 / 8$ & $9 / 1(7 / 1)$ \\
\hline (Orense) & Sta. Ouxea & 42 & 20 & 7 & 55 & 110 & 10 & -7.7 & $1 / 3-13 / 10$ & $23 / 12(27 / 12)$ \\
\hline Pazoo & S. Martín & 42 & 10 & 7 & 49 & $100^{1 / 4}$ & 3 & -5.7 & $6 / 3-8 / 10$ & $11 / 11-20 / 3$ \\
\hline Celanova & S. Miguel & 42 & 9 & 7 & 57 & $90^{3 / 4}$ & 2 & 0.6 & $22 / 3-21 / 9$ & $29 / 9$ \\
\hline Xinzo & S. Mariña & 42 & 3 & 7 & 43 & $112^{1 / 4}$ & $03 / 4$ & -16.1 & $4 / 2-7 / 11$ & $18 / 7$ \\
\hline Mosteiro & ------ & 41 & 59 & 7 & 47 & $881 / 4$ & $-01 / 2$ & 0.5 & $21 / 3-22 / 9$ & ------ \\
\hline Mixós & S. María & 41 & 57 & 7 & 27 & $65 \frac{1 / 4}{4}$ & $31 / 2$ & 20.3 & $22 / 5-21 / 7$ & $1 / 1-19 / 5-15 / 8-8 / 12$ \\
\hline
\end{tabular}

en realidad, pues para cada posición en el horizonte hay dos días en que el sol puede salir o ponerse por él), pero que será diferente, para esos mismos valores, si nos desplazamos a otro emplazamiento con otra latitud. Sería pues difícil y engorrosa la comparación de las orientaciones de las iglesias de nuestra muestra. Para ayudar en esta comparación utilizamos la declinación astronómica. Esta coordenada se define como la proyección en la esfera celeste de la coordenada de la latitud terrestre y se obtiene a partir de una sencilla combinación del azimut, la altura del horizonte y la latitud (para una definición concreta y mayor discusión sobre su uso véase, e.g. Belmonte \& González-García 2013). Esta nueva coordenada es independiente de la latitud terrestre y nos permitirá comparar todas las iglesias de nuestra muestra entre sí, así como con los movimientos del sol a lo largo del año.

Estos movimientos presentan dos extremos en la declinación en valores de $\pm 231^{1}{ }^{\circ}$ actualmente. Estos valores extremos del sol ocurren en los solsticios de verano (máxima declinación) e invierno (mínima, para el hemisferio norte), mientras que para los equinoccios astronómicos, por definición, será cuando la declinación sea de $0^{\circ}$. Entre el equinoccio de Primavera y el de Otoño el sol presentará declinaciones positivas, mientras que entre el equinoccio de Otoño y el de Primavera serán negativas. Por medio de la ecuación del tiempo o provistos con un programa de planetario, es relativamente sencillo traducir la declinación del sol en fechas tanto del calendario Gregoriano proléptico, es decir, del calendario que usamos nosotros actualmente, que sigue de forma bastante fiel los movimientos del sol en las estaciones (año trópico), o en fechas del calendario Juliano, en uso durante la época de construcción de los templos investigados en este artículo y que acumula un desfase con respecto al año trópico de un día cada 128 años aproximadamente.

\section{MUESTRA}

El número y tipo de iglesias prerrománicas en Galicia o para las cuales existe una fase que puede 
ser identificada como prerrománica es alto (SÁNCHEZ PARDO, 2012) . El número de estas iglesias es variado, ya que depende en gran medida del estado de conservación. Hay un gran número de iglesias que presentan actualmente varias fases de construcción diferentes, y las etapas más antiguas son comúnmente reutilizadas. Así que nuestro criterio para seleccionar los templos usados en nuestra muestra ha sido que al menos la línea de base de la iglesia fuera reconocible como del periodo prerrománico. En varios casos, esto es relativamente fácil, ya que algunos templos han sufrido restauraciones completas en el pasado reciente, mientras que en otros es más difícil de discernir. Algunas iglesias presentan varias fases constructivas con cambios de orientaciones entre ellas. Éstos a veces se incorporan en un solo edificio, que muestra un cambio en la orientación, o una nueva iglesia a menudo se construye en el sitio de una anterior, destruido durante la invasión musulmana o por otras causas, como la Catedral de Santiago de Compostela.

La Tabla 1 presenta la muestra completa de datos en este momento. Se compone de 25 iglesias para Galicia. La muestra no está completa y estamos actualmente trabajando en tomar más datos en diferentes zonas de Galicia, ya que actualmente tenemos una buena muestra en áreas como Ourense, pero es aun escasa en otras (véase la Figura 2). Sin embargo, esta muestra ya permite extraer una serie de conclusiones, así como su comparación con el trabajo para el resto de la Península de González-García \& Belmonte (2015) o de toda Europa (GONZÁLEZ-GARCÍA 2014).

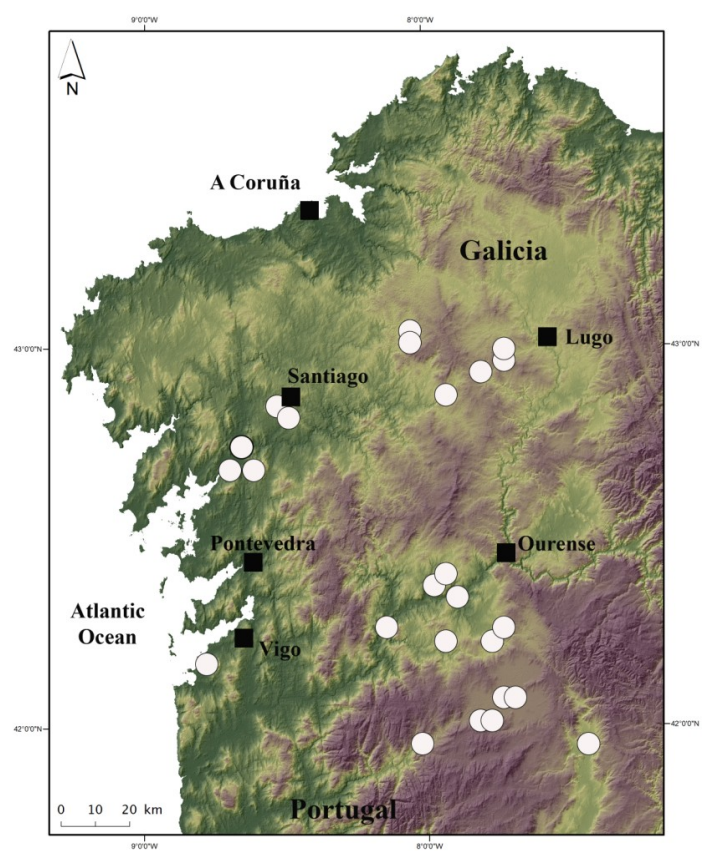

Fig. 2. Situación de las 25 Iglesias prerrománicas gallegas medidas hasta el momento.

Fig. 2. Geographical location of the 25 pre-Romanesque churches measured in Galicia.
En la Tabla 1, la primera columna identifica el sitio donde está situada la iglesia, mientras que la segunda nos dice el nombre de la iglesia, si este es conocido. Las cuatro columnas siguientes nos dan la situación, en coordenadas de latitud y longitud. Las dos columnas siguientes dan nuestros datos del trabajo de campo, el azimut y la altura del horizonte en la dirección hacia la que apunta el ábside de la iglesia, debidamente corregidos. La siguiente columna da la declinación astronómica correspondiente, mientras que las dos últimas columnas indican las fechas correspondientes de salida (o puesta en el caso de que el ábside mirase a poniente) del sol en el calendario Gregoriano, y la fecha de celebración del santo si éste es conocido.

\section{RESULTADOS}

La Figura 3 muestra el diagrama de orientación de las 25 iglesias de nuestra muestra. En este diagrama la orientación de cada iglesia está indicada por una línea corta en el interior del círculo. Este círculo es una representación del horizonte, donde se marcan los puntos cardinales y los lugares de salida y puesta del Sol en los extremos solsticiales. Esta figura indica que todas nuestras iglesias están dentro del rango de salidas o puestas solares. Solo hay una iglesia (Ouvigo) que presenta su orientación hacia el sector occidental, mientras que el resto (24) se orientan hacia el oriental. Parece existir una concentración de orientaciones hacia el este y una tendencia a evitar los solsticios.

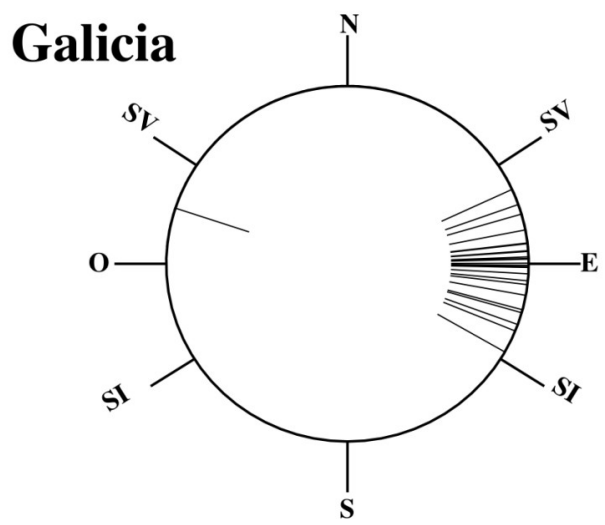

Fig. 3. Diagrama de orientación de las 25 iglesias prerrománicas de nuestra muestra. La orientación del ábside de cada iglesia está indicada por una línea continua dentro del círculo, que representa el horizonte. Las marcas exteriores muestran los cuatro puntos cardinales, así como los puntos extremos de salida y puesta del sol en el Solsticio de verano (SS) y en el de invierno (WS).

Fig. 3. Orientation diagram for the 25 pre-Romanesque churches in our sample. The orientation of the apse is indicated by a short solid line inside the circle. The marks outside the circle indicate the cardinal directions and the extreme postions of sunrise and sunset for the solstices. 
Esta impresión queda ratificada si nos fijamos en el histograma de orientaciones de la Figura 4.

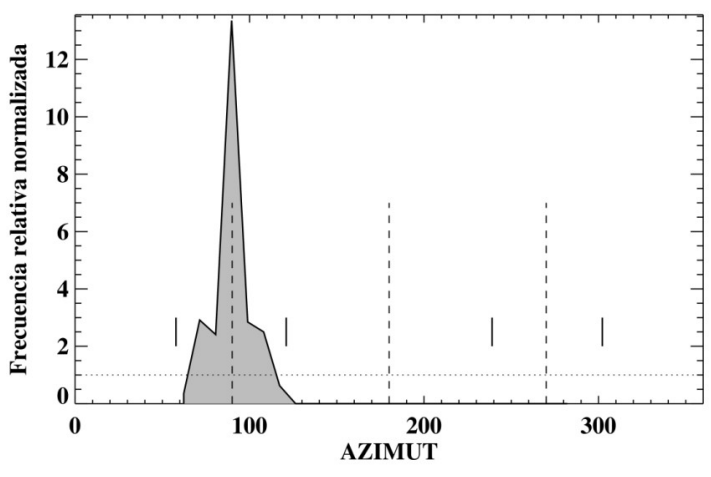

Fig. 4. Histograma normalizado por la media de las orientaciones de las 25 iglesias de nuestra muestra. Las líneas verticales a trazos muestran los puntos cardinales, siendo el 0 el norte. Las líneas continuas verticales muestran los extremos de salida y puesta del sol.

Fig. 4. Histogram showing the frequency, normalized by the mean of the 25 churches in our sample. The dashed vertical lines indicate the cardinal directions and the short solid lines the solstices.

En este histograma y el siguiente (Figura 5) hemos utilizado un suavizado por una función llamada 'kernel' para producir la distribución de densidad (en adelante KDD) (véase, por ejemplo, González-García y Belmonte 2014b). En cada entrada, ya sea en azimut o en declinación, se multiplica el valor del número de ocurrencias de un valor por el kernel con paso de banda dado. Para este proceso, hemos usado un kernel de Epanechnikov con un ancho de banda de dos veces el error estimado en declinación. Para ser capaz de decir si una medida es significativa, se utiliza una frecuencia relativa normalizada para escalar nuestras KDDs o histogramas. Para ello, se divide el número de ocurrencias de un valor dado por el número medio de veces que ocurrió esa muestra; esto es equivalente a la comparación con los resultados de una distribución uniforme del mismo tamaño que la muestra de datos, y con un valor igual a la media de los datos.

El histograma de la Figura 4 nos ratifica que la mayor parte de las orientaciones están muy bien centradas en torno al este, siendo 13 veces más probable encontrar una iglesia de nuestra muestra orientada hacia el este que con cualquier otra orientación.

Otro dato que podemos extraer del histograma, y que ya veíamos en el diagrama de orientaciones, es que no existe ninguna iglesia orientada hacia los solsticios, ya sea de verano o invierno.

Sin embargo, dado que las iglesias que hemos medido están situadas en terrenos donde el horizonte dista de ser plano y además estamos considerando iglesias en un rango apreciable de latitudes, conviene corroborar estas impresiones estudiando el histograma de declinaciones (Figura 5). En este caso, el histograma está normalizado, restando a

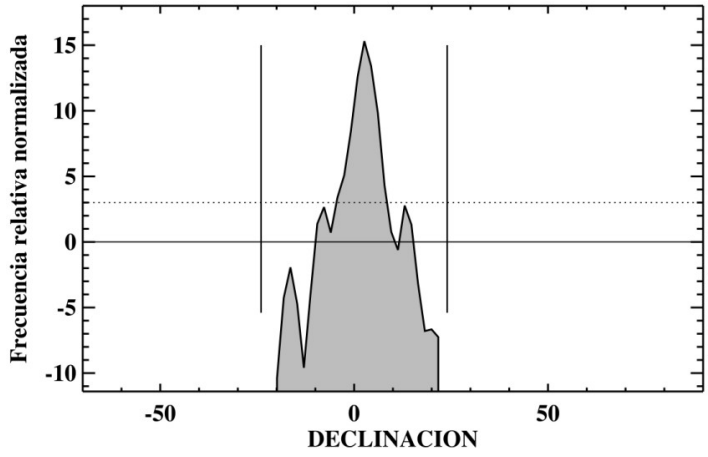

Fig. 5. Histograma de declinaciones normalizado. Las líneas verticales continuas muestran el rango de declinaciones solar. La línea horizontal punteada muestra el valor 3. Para más detalles, véase el texto.

Fig. 5. Normalized declination histogram. The vertical lines indicate the solar range, The dotted horizontal line indicates a value of 3 . For details, see text.

cada valor de declinación el valor promedio de la propia distribución y dividiendo la resultante por la desviación estándar de esta distribución. De esta manera, el eje de ordenadas nos da cuan significativo por encima de esa desviación estándar es un máximo que encontremos. Es común en ciencias naturales usar que un pico es significativo si está por encima de 3 veces ese valor, aunque en ciencias humanas esto a veces se relaja a considerarlo dos veces por encima.

En la figura 5 solo encontramos un máximo por encima de 3 sigma. Es para un valor de la declinación de $2.6^{\circ}$. Este valor está bastante cerca del valor del equinoccio astronómico, $0^{\circ}$. Por encima de dos sigma aparecen otros dos máximos, para valores de declinación $13.0^{\circ} \mathrm{y}-7.8^{\circ}$.

El valor de $2.6^{\circ}$ corresponde a la declinación solar para el 27 de Marzo o el 16 de Septiembre en el calendario Gregoriano Proléptico. Para el calendario Juliano, correspondería con el 25 de Marzo o 14 de Septiembre para el año 500 AD. Para el año 1000 correspondería al 21 de Marzo o al 10 de Septiembre.

El valor de $13.0^{\circ}$ corresponde al 26 de Abril o el 17 de Agosto en el Calendario Gregoriano proléptico, o al 24 de Abril o 15 de Agosto del 500 AD. Correspondería al 21 de Abril o 12 de Agosto del 1000 AD.

Finalmente, el valor de $-7.8^{\circ}$ equivaldría al 1 de Marzo o el 13 de Octubre en el calendario Gregoriano proléptico o al 27 de Febrero o 10 de Octubre del $500 \mathrm{AD}$ o el 24-25 de Febrero y el 6 de Octubre del año 1000.

\section{DISCUSIÓN}

No tenemos aun la muestra completa y además, el tema de la cronología y periodización de las iglesias prerrománicas es complejo, por lo que la discusión por períodos la dejamos para cuando tengamos la muestra completa. Vamos a continuación a discutir los resultados generales hasta ahora obtenidos. 
En primer lugar, comparando los datos de las dos últimas columnas de la tabla 1 es aparente que no hay ninguna iglesia para la cual la orientación del ábside coincida con la festividad del santo patrón, ni siquiera teniendo en cuenta el desfase entre calendario Juliano y Gregoriano. Un caso a destacar, sin embargo, es el de la basílica de la Ascensión de Augasantas, que sería compatible en caso de considerar la orientación del pie de la iglesia en lugar de la cabecera (véase García Quintela et al. 2014). Esto abre una posibilidad a explorar en el futuro en otras iglesias.

Es interesante que la única iglesia que tiene una orientación a poniente (Ouvigo) sea una de las que tienen adscrita una más temprana edificación, siendo posiblemente de época paleocristiana (Sánchez Pardo 2012). Recientemente se ha establecido que la cripta de la basílica de la Ascensión de Augasantas (Ourense) presenta varias fases, comenzando como una sauna (posible monumento con forno) reformado posiblemente hacia el siglo VI, tal vez ya para dar lugar a una capilla cristiana (BLANCO ROTEA et al. 2009a.) En tal caso la orientación de tal capilla debería posiblemente considerarse hacia poniente, funcionando el horno de la sauna como el posible ábside de la capilla. Otro caso interesante es el de Santa Eulalia de Bóveda. Si bien el edificio singular se ha interpretado tradicionalmente como un posible santuario romano, recientemente se ha abierto una nueva línea de interpretación según la cual podría ser un edificio altomedieval o tardoantiguo pero no romano y por tanto tal vez relacionado con el culto cristiano (Blanco Rotea et al. 2009b). En tal caso, su orientación, esencialmente la misma que la de la iglesia asturiana superior, se dirigiría a $180^{\circ}$ respecto a esta, hacia poniente. Según veíamos en la introducción y como muestra González-García (2015), no es extraño encontrar iglesias orientadas hacia poniente para esta época.

En cualquier caso, la mayor parte de las iglesias prerrománicas de Galicia parece corresponder con la idea de la orientación canónica hacia el equinoccio. De hecho hemos visto que es varias veces más probable encontrar una orientación equinoccial que de otro tipo en nuestra muestra. Otra cosa es saber si este máximo se corresponde con la orientación hacia el equinoccio astronómico, el equinoccio definido como el orto del 21 de Marzo o el del día de la Anunciación (25 de Marzo).

El primero, puede quedar descartado, pues la sistematicidad de la desviación del máximo que aparece en la Figura 5 respecto al valor del equinoccio astronómico $\left(0^{\circ}\right)$, está fuera de duda, dado el número de iglesias que aparecen en él y el error de las medidas.

Si nos fijamos en los valores de las fechas que corresponderían con ese máximo, parece clara la intencionalidad hacia uno de los dos valores en Marzo o bien el 21 o bien el 25. Es interesante la dicotomía, pues no es la primera vez que aparece, si bien lo más normal es ver que este máximo se corresponda al 25 de marzo. Al menos así parece ser en el resto de la Península, donde además parece existir una tendencia con el tiempo, de forma que este máximo se va desplazando hacia valores de declinación mayores según las épocas (véase González-García \& Belmonte 2015). No es posible realizar este ejercicio en nuestra muestra actualmente, salvo para la catedral de Santiago (ver infra). Los motivos son dos: el primero es la escasez de datos. A pesar de que 25 es un número importante de iglesias, no es suficiente para realizar un estudio veraz dividiendo en submuestras. El segundo es la gran dificultad, salvo para un número contado de ocasiones, de determinar de forma certera la época de construcción de nuestras iglesias.

Sin embargo, sí que es cierto que el máximo de nuestra distribución de la Figura 5, presenta una anchura apreciable. Si realizamos el ejercicio de disminuir la achura del paso de banda, podemos ver como este máximo se puede desdoblar en dos máximos principales, el más poblado para un valor de declinación de $4.2^{\circ} \mathrm{y}$ un segundo para $0.9^{\circ}$. El primero correspondería a la fecha del 25 de Marzo para el entorno del año 1000AD. Así pues, es posible que ese máximo esté enmascarando un proceso de orientación hacia el equinoccio a partir del 25 de Marzo que debido a la naturaleza errante del calendario Juliano respecto a las estaciones, se va desplazando con el tiempo, dando como resultado un máximo principal muy ancho en la Figura 5.

A este respecto es interesante destacar que el valor de la orientación de la basílica de Santiago construida por Alfonso III, que es la que se presenta en esta muestra, concuerda con tal fecha. La orientación de esta basílica es ligeramente diferente que la de la catedral románica posterior, como ya hace notar Guerra Campos (1982). Lo interesante es que la orientación de la catedral sería compatible de nuevo con la fecha del 25 de Marzo para las fechas de erección de la catedral románica varios siglos después, explicando la diferencia de orientación por la naturaleza errante del calendario Juliano respecto al año trópico. Sin embargo, con la muestra actual no podemos descartar que de forma complementaria para otras iglesias se utilizase en Galicia el 21 de Marzo.

Es pues necesario ampliar la muestra a fin de determinar esto, así como ajustar lo mejor posible los períodos de construcción que permitan aclarar esta cuestión.

Respecto a los dos picos con significancia por encima de 2 sigma, es interesante que el que aparece en valores de declinación en torno a $14^{\circ}$ es el mismo que da el máximo de declinaciones para el período asturiano en las 15 iglesias asturianas consideradas por González-García \& Belmonte (2015). Sería pues muy conveniente ver si esas iglesias tienen cronologías que correspondan con 
este período, como parece podría ser el caso para la de San Xes de Francelos. En cuanto a las fechas que corresponden con esas orientaciones, parece significativo el que el orto de Agosto esté cercano a una fecha mariana importante como es la del 15 de Agosto, la Asunción. Sería interesante ver si este aparente interés se corrobora con alguna noticia en las crónicas o en la epigrafía de la época.

Finalmente el tercer máximo ocurre para declinaciones de $-7.8^{\circ}$ y para fechas entorno a Finales de Febrero o mediados de Octubre. No tenemos constancia de ninguna fiesta singularmente importante en estas fechas para esta época y por tanto queda abierto el interrogante hasta que tengamos una muestra más amplia que seguro ayudará a discernir este caso. En este sentido, una idea a explorar en el futuro es si algunas de estas fechas pueden estar asociadas a algún tipo de calendario agrícola o tradicional.

\section{CONCLUSIONES}

A partir de la medida de las orientaciones de 25 iglesias prerrománicas en Galicia podemos avanzar un número de conclusiones preliminares.

Es claro el interés de los constructores en orientar las iglesias de forma canónica según las prescripciones de la Iglesia, apareciendo una gran mayoría con orientaciones muy cercanas al equinoccio. Este muy posiblemente se determinase como el 25 de Marzo, si bien es necesario ampliar la muestra para constatar esto con mayor certidumbre.

Un número importante de iglesias tienen orientaciones que las haría compatibles con las que aparecen entre las iglesias del período asturiano. A este respecto, hay que tener en cuenta que algunas iglesias de posible cronología asturiana, como la de Bóveda o las dos de Santiago, si bien no tienen la orientación más característica de las asturianas, su orientación no sería rara entre ellas.

En cualquier caso, este es un estudio preliminar y será necesario un mayor número de medidas en el resto de iglesias con fases prerrománicas de la región a fin de afinar estas conclusiones.

\section{AGRADECIMIENTOS}

La mayor parte de los datos del trabajo de campo aquí expuestos se han beneficiado de la ayuda y colaboración con numerosas personas. Me gustaría agradecer sobretodo a M.V. García Quintela, Rebeca Blanco Rotea, Sonia García Rodríguez, Yolanda Seoane Veiga y José Carlos Sánchez Pardo. Querría agradecer también a los evaluadores del artículo por sus comentarios que han ayudado a mejorar la calidad del mismo. ACGG es Ramón y Cajal del MINECO.

\section{BIBLIOGRAFÍA}

Belmonte, J.A., GonzÁlez-García, A.C. 2013. Metodología y fundamento de las observaciones arqueoastronómicas. En: F. Criado-Boado, A. MartínezCortizas, M.V. García Quintela (eds), Petroglifos, paleoambiente y paisaje. Estudios interdisciplinares de arte rupestre de Campo Lameiro (Pontevedra) Traballos de Arqueoloxía e Patrimonio 42. 59-63.
Blanco-RoteA, R. MAÑANA-BorrazÁs, P., MATOFresÁn, C., Rodríguez-Costas, A. 2009. La basílica de la Ascensión y Os Fornos (Allariz, Ourense). Aquae Flaviae, 41: 467-478.

Blanco-Rotea, R., Benavides García, R., SAnjurjo SÁncheZ, J., Fernández Mosquera, D. 2009. Evolución constructiva de Santa Eulalia de Bóveda (Lugo, Galicia). Arqueología de la Arquitectura, 6: 149-198.

Chauncey, H. 1700. The Historical Antiquities of Hertfordshire. London.

ČAVAL, S. 2009. Astronomical orientations of Sacred Architecture during the Medieval period in Slovenia. En: J.A. Rubiño-Martín, J.A. Belmonte, F. Prada and A. Alberdi (eds.), Cosmology Across Cultures, San Francisco, Astronomical Society of the Pacific: 209-19.

Delgado-Gómez, J. 2006. El porqué de la orientación de las iglesias. Lucensia, 16, 33: 347-56.

Esteban, C., Belmonte, J.A., Perera Betancort, M.A., MARrero, R., Jimenez GonZALEZ, J.J. 2001. Orientations of pre-Islamic temples in North-West Africa. Archaeoastronomy, 26: S65-84.

Gangui, A., González-García, A.C., Perera BetanCORT, M.A. AND BELMONTE J.A. 2015. La orientación como una seña de identidad cultural: Las Iglesias históricas de Lanzarote. Tabona, en prensa.

García Quintela, M.V., GonZÁlez-García, A.C. AND SEOANe-VeIgA, Y. 2014. De los Solsticios en los castros a los santos cristianos; la creación de los paisajes cristianos en Galicia. Madrider Mitteilungen, 55: 443-485.

GONZÁLEZ-GARcíA, A.C. 2015. A voyage of Christian medieval astronomy: symbolic, ritual and politica orientation of churches. En: F. Pimenta, N. Ribeiro, F. Silva, N. Campion, A. Joaquinito and L. Tirapicos (eds.), Stars and Stones. British Archaeology Reports, in press

GonZÁlez-GarcíA, A.C., Belmonte J.A. 2006. Which Equinox? Archaeoastronomy, The Journal of Astronomy in Culture, XX: 97-107.

GonZÁlez-GARCíA, A.C., BElmonte J.A. 2014a. Interactions Between Islamic and Christian Traditions in the Iberian Peninsula. En: C.L.N. Ruggles (ed.), Handbook of Archaeoastronomy and Ethnoastronomy, Nueva Yor, Springer: 1695-1702.

GonZÁlez-GarcíA, A.C., Belmonte J.A. 2014b. Sacred architecture orientation across the Mediterranean: a comparative statistical analysis. Mediterranean Archaeology and Archaeometry, 14 (2): 95-113.

GonzÁlez-GarcíA, A.C., Belmonte J.A. AND CostAFERRER, L. 2015. The orientation of preRomanesque churches in Spain: Asturias, a case of power re-affirmation. En: M.A. Rappenglueck, B. Rappenglueck and N. Campion (eds.), Astronomy and Power. British Archaeology Reports, in press.

GonZÁlez-GARCÍA, A.C., BELMONTE J.A. 2015. The Orientation of Pre-Romanesque Churches in the Iberian Peninsula. Nexus Network Journal, 17: DOI: 10.1007/s00004-014-0231-7

Godoy Fernandez, C. 2004. A los pies del templo. Espacios litúrgicos en contraposición al altar: una revision. Antigüedad Cristiana XXI: 473-89. 
GUERRA CAMPOS, J. 1982. Exploraciones Arqueológicas en Torno al Sepulcro del Apostol Santiago. Burgos. Cabildo de la Catedral de Santiago.

Jonhson, W. 1912. Byways in British Archaeology, Cambridge, Cambridge University Press.

MCCluSKey, S.C. 1998. Astronomies and cultures in early Medieval Europe, Cambridge, Cambridge University Press.

MCCluSKEy, S.C. 2004. Astronomy, Time, and Churches in the Early Middle Ages, En: M.-T. Zenner, Villard's legacy: Studies in Medieval Technology, Science and Art in Memory of Jean Gimpel. Ashgate: Aldeshot: 197-210.

MCCluskey, S.C. 2010. Calendric cycles, the eighth day of the World and the orientation of English Churches, En: C. Ruggles and G. Urton (eds.), Skywatching in the Ancient World, New Perspectives in Cultural Astronomy. Bolder, University Press of Colorado: 331-353.
NisSEN, H. 1906. Orientations. Studien zur Geschichte der Religion. Berlin, Weidmann.

PÉreZ-VAlCARCEL, J. 1998. La orientación de las iglesias del Camino de Santiago, En: F. Bores, J. Fernández, S. Huerta, E. Rabasa, Actas del Segundo Congreso Nacional de Historia de la Construcción, La Coruña, Servicio de Publicaciones Universidad de La Coruña: 391-396.

SÁNCHEZ PARDO, J.C. 2012. Arqueología de las Iglesias Tardoantiguas en Galicia (SS. V-VIII). Una Valoración de Conjunto. Hortus Artium Mediavalium, 18/2: 395-414.

RugGles, C. L. N. 1999. Whose equinox? Archaeoastrono$m y, 22: \mathrm{S} 45-50$.

Vogel, C. 1962. Sol aequinoctialis. Problemes et tecnique de l'orientation dans le culture chretien. Revue Sciences Religieuses, 36: 175-211. 